\title{
Effects of proximal unconditioned stimulus preexposure on ingestional aversions learned as a result of taste presentation following drug treatment
}

\author{
MICHAEL DOMJAN \\ University of Texas at Austin, Austin, Texas 78712
}

\begin{abstract}
Taste aversions were conditioned by exposing subjects to a $1.0 \%$ saccharin solution $30 \mathrm{~min}$ after an injection of lithium chloride. The aversion learning was disrupted if subjects had also received an additional lithium injection some time earlier (Experiments 1-3). This interference effect of US preexposure was a decreasing function of the preexposure interval, beyond the optimal interval $(105 \mathrm{~min})$ for observing the phenomenon (Experiment 1), and was directly related to the dose of the preexposure injection (Experiment 2). No interference with conditioning occurred at short (e.g., 30-min) preexposure intervals (Experiment 1), probably because under these circumstances the preexposure injection itself conditioned a strong aversion (Experiment 4). At moderate $(105-\mathrm{min})$ but not at short $(30-\mathrm{min})$ preexposure intervals, the interference with aversions learned as a result of taste exposure following drug injection was comparable to the interference with learning in a more conventional forward conditioning procedure (Experiments 3 and 4). These findings are similar to previously documented effects of proximal CS- and US-preexposure and are consistent with recent stimulus rehearsal and opponent-process theories.
\end{abstract}

The extent to which one stimulus becomes associated with another depends not only on the temporal sequence and schedule of their presentation, but also on the nature of the stimulus events. One stimulus characteristic that is important for the establishment of associations is novelty. Reductions in the novelty of conditioned (CS) and unconditioned (US) stimuli have been observed to reduce their associability in a variety of situations (e.g., Lubow, 1973; Siegel \& Domjan, 1971). This interference effect of CS and US preexposure is especially evident in ingestional aversion learning. Numerous studies have shown that exposure to either the CS flavor or the US malaise before a conditioning trial attenuates aversion learning. These experiments usually have involved repeated preconditioning presentations of the CS or US event, with at least $24 \mathrm{~h}$ between successive preexposures and at least $24 \mathrm{~h}$ between the end of the preexposure phase and the subsequent conditioning trial (e.g., Cappell \& Le Blanc, 1975; Domjan, 1972; Riley, Jacobs, \& LoLordo, 1976; Vogel \& Nathan, 1976). Since, typically, 24 h or more intervened between preexposure and conditioning, the resultant interference effects were produced by stim-

The research was supported by Grant BNS 77-01552 from the National Science Foundation. The technical assistance of Tracy McDonald and Mark Stanton is gratefully acknowledged. Requests for reprints should be addressed to Michael Domjan, Department of Psychology, University of Texas, Austin, Texas 78712. ulus preexposures which may be characterized as remote from the conditioning trial.

In contrast to the experiments described above, recent research (Best \& Gemberling, 1977; Domjan \& Best, 1977) has shown that CS and US preexposures closer to the conditioning trial can also disrupt aversion learning. In one of these studies (Best \& Gemberling, 1977), maximal interference with conditioning occurred when subjects were exposed to the CS flavor $4 \mathrm{~h}$ before the conditioning trial, with more remote CS preexposures having less of a deleterious effect. In the other experiment (Domjan \& Best, 1977), US exposure $30 \mathrm{~min}$ before conditioning produced more disruption of conditioning than US exposure $6 \mathrm{~h}, 1$ day, or 2 days before conditioning. These findings suggest that, in addition to the long-lasting interference effects observed following repeated remote CS and US preexposures, disruption of conditioning can also occur as a result of a single preconditioning exposure to either the CS or the US much closer to the conditioning trial. These latter effects may be attributed to proximal CS and US preexposure.

The interference effects of CS and US preexposure that are only evident for a limited period following preexposure are no doubt mediated by temporary changes which follow stimulus exposures. The recent information processing model proposed by Wagner (1976) provides a mechanism for such effects. According to this model, a stimulus will influence behavior to the extent that it is rehearsed in short-term memory, and 
less rehearsal will be generated by a stimulus presentation if that stimulus is already available or primed in short-term memory. The proximal CS- and USpreexposure phenomena are attributed to decreased CS and US effectiveness during the conditioning trial due to the priming of the CS and US in short-term memory when the conditioning presentation of these events occurs. The temporary nature of the interference effects is attributed to the loss with time of the preexposure stimulus from short-term memory.

Wagner's (1976) rehearsal theory provides a parsimonious account of both the proximal CS- and USpreexposure effects. However, because the procedures used to demonstrate the two phenomena in the tasteaversion paradigm have involved different stimulus sequences, it is possible that different processes may be responsible for each effect. Each phenomenon has been investigated only with forward conditioning procedures in which presentation of the CS flavor is followed by the US. Therefore, the stimulus sequence used in proximal CS-preexposure experiments (Best \& Gemberling, 1977) has been CS-CS-US, whereas the stimulus order employed in proximal US-preexposure experiments (Domjan \& Best, 1977) has been US-CS-US. Because of this difference in the sequence of CS and US presentations, one could argue that the rehearsal theory is more applicable to the proximal CS-preexposure effect than to the proximal US-preexposure effect. In the CSpreexposure procedure (CS-CS-US), the two repeated events (CSs) are not separated by a novel stimulus which may compete for the limited capacity of shortterm memory. Therefore, rehearsal stimulated by the first presentation of the CS could very well be taking place when the CS is again presented shortly thereafter. In contrast, with proximal US preexposure (US-CS-US), rehearsal of the repeated event (this time the US) may be interrupted by presentation of the novel CS during the conditioning trial, and this might make it less likely that the interference effect is due to priming of the US in short-term memory.

The interference produced by presentation of the US shortly before a CS-US pairing (Domjan \& Best, 1977) can be accommodated by interpretations other than Wagner's (1976) rehearsal theory. The US-CS-US stimulus sequence differs from the procedure administered to nonpreexposed control subjects (CS-US) not only in that the US is presented twice, but also in that the CS is presented after the US. Therefore, the attenuation of conditioning observed may be due to the presentation of the CS after the preexposure US rather than to the repetition of the US, as assumed by rehearsal theory. Presentation of the CS after the preexposure US may result in reduced aversion performance because the preexposure US alters the perception of the CS and thereby contributes to a generalization decrement when subjects are subsequently tested for their aversion to the $\mathrm{CS}$ in the normal state. Alternatively, post-US presentation of the CS may interfere with aversion learning because the CS is paired with recovery. from the malaise induced by the preexposure US.

The above speculations suggest that the deleterious effects of proximal US preexposure may be evident only in a comparison between a preexposed group which receives contact with the CS during conditioning following exposure to the preexposure US (US-CS-US) and a control group which receives its conditioning exposure to the CS in the normal state (CS-US). This does not appear to be the case in rabbit eyelid conditioning. In that preparation, US priming has been found to disrupt conditioning even if the priming presentation of the US is administered after the CS (Terry, 1976, Experiment 4). Furthermore, presentation of the priming US before the CS is not sufficient to interfere with eyelid conditioning because the deleterious effects of such a priming US can be mitigated by presenting a disruptive event which displaces the priming US from short-term memory (Terry, 1976, Experiment 2).

The present experiments were designed to determine whether or not demonstration of the proximal USpreexposure effect in taste-aversion learning is dependent on a comparison between subjects which receive their contact with the CS following exposure to the preexposure US (US-CS-US) and subjects which receive their contact with the CS in the normal state (CS-US). In contrast to previous proximal US preexposure studies in which flavor aversions were conditioned by injecting subjects with the toxin lithium after presentation of the CS flavor (CS-Li) (Domjan \& Best, 1977), in the present experiments flavor aversions were conditioned by injecting subjects with lithium before the CS presentation (Li-CS).

Initial efforts to condition flavor aversions with lithium injected before the flavor presentation met with little success (Barker \& Smith, 1974). However, recent studies employing more concentrated taste solutions have reported significant aversions learned to $.5 \%$ saccharin presented 30 min after lithium injection (Domjan, Schorr, \& Best, 1977) and 1.0\% saccharin presented $60 \mathrm{~min}$ after lithium treatment (Domjan \& Gregg, 1977). Furthermore, unpaired taste-lithium control groups showed that these aversions reflected associative rather than sensitization processes.

Subjects in the present experiments were conditioned to avoid a $1.0 \%$ solution of saccharin by receiving an injection of lithium $30 \mathrm{~min}$ before the saccharin exposure. US preexposure was accomplished by the administration of two lithium injections, one $30 \mathrm{~min}$ before taste exposure and the other some time earlier. Thus, both control and preexposed subjects received their conditioning presentation of the CS flavor $30 \mathrm{~min}$ after lithium treatment. Since lithium-induced suppressions of saccharin (Barker \& Smith, 1974; Domjan, 1977) and water (Barker \& Smith, 1974) intake have been observed within $15 \mathrm{~min}$ after drug injection, both 
control and preexposed subjects no doubt received access to the CS after the start of lithium malaise.

\section{EXPERIMENT 1}

Experiment 1 was designed to determine whether or not a single preconditioning US exposure can attenuate flavor aversions which are learned as a result of postinjection taste presentation. The preexposure-US treatment was administered at various times to independent groups so that the time course of the interference effect could also be ascertained.

\section{Method \\ Fifty-nine male and 33 female 50- to 60 day-old Sprague- Dawley rats born in the laboratory were individually housed in wire-mesh hanging cages with continuous access to Purina Rat Chow throughout the experiment. Light days before condition- ing, access to water was restricted to $30 \mathrm{~min}$ daily. The subjects were assigned 10 nine groups made as similar as possible in dis- tribution of weights and gender. On the conditioning day, seven of the nine groups received two $1.8 \cdot \mathrm{m}$ eq $/ \mathrm{kg}$ intraperitoneal in. jections of .15-M lithium chloride. The second injection was fol- lowed 30 min later by access to a $1.0 \%$ (wcight/volume) solution of sodium saccharin for $32.5 \mathrm{~min}$ or until the subjects drank $1 \mathrm{ml}$, whichever occurred first. For Groups $30(\mathrm{~N}=10), 70(\mathrm{~N}=10)$. $105(N=10), 140(N=10), 240(N=11)$, and $400(N=10)$. the first lithium injection was administered $30,70,105,140$, 240, and $400 \mathrm{~min}$ before their second injection with the drug; for Group 3-Day $(\mathrm{N}=10)$, the first lithium injection occurred 3 days before the second. In contrast to the various groups that recejved two lithium injections, the taste-aversion control group. TAC $(N=11)$, was not preexposed to the US and re- ceived only one lithium injection $30 \mathrm{~min}$ before the saccharin presentation. Group No-AV $(\mathrm{N}=10)$ served as a nonconditioned (no-aversion) control group and was injected with $1.8 \mathrm{meq} / \mathrm{kg}$ physiological saline $30 \mathrm{~min}$ before saccharin access. On the con- ditioning diy, the subjects received their daily 30-min access to water $1.5 .3 .5 \mathrm{~h}$ after the saccharin exposure. The order in which the various groups were conditioned was unrelated to the US- preexposure interval and was varied between the two replica- tions which constituted the experiment. Therefore, there was no systematic relationship between the US-preexposure interval and when subjects received their maintenance access to water on the conditioning day. Five to 6 days after conditioning. each subject received a 60 -min one-bottle test with the saccharin solu. tion in the home cage following $23.5 \mathrm{~h}$ water deprivation.}

\section{Results}

Mean intakes of saccharin for Groups TAC, 30, 70, $105,140,240,400,3$-Day, and No-Av during the con. ditioning trial were $.6, .6, .3, .4, .5, .7, .9, .6$, and $1.0 \mathrm{ml}$. respectively. A one-way analysis of variance computed on these data revealed a significant effect of Groups $[F(8,83)=7.2, p<.01]$. Subsequent comparisons with the Newman-Keuls test $(\mathrm{p}<.05)$ showed that Group No-Av drank more saccharin during conditioning than every other group except Group 400. Group 400 also drank significantly more than Groups TAC, 30, 70, 105 , and 140 , and Group 240 drank significantly more than Group 70. No other two-group comparisons were statistically significant.

The amount of saccharin each group consumed during the postconditioning test is summarized in Figure 1. As expected, subjects which had not been injected

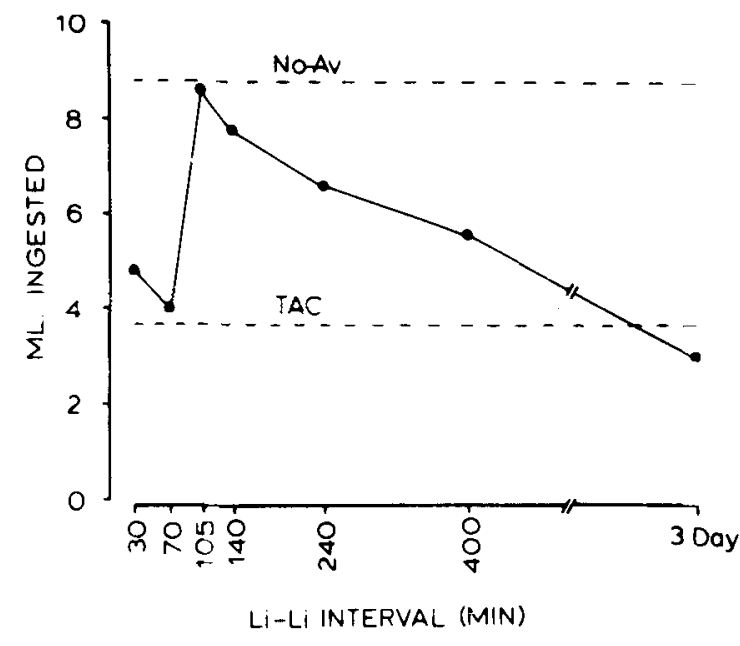

Figure 1. Mean saccharin intakes of independent groups which previously received two lithium injections at various intervals, followed $30 \mathrm{~min}$ later by exposure to saccharin. Group TAC previously received only one lithium injection, $30 \mathrm{~min}$ before saccharin exposure, and Group No-Av did not have saccharin paired with lithium.

with lithium on the conditioning day (Group No-Av) drank the most saccharin. In contrast, the taste-aversion control group, TAC, and Group 3-Day, which was preexposed to lithium 3 days before conditioning, showed the strongest saccharin aversions during the test session. The mean intakes of each of the other US preexposed groups were between these two extremes. A substantial interference with aversion learning was evident if the US preexposure occurred $105 \mathrm{~min}$ before conditioning, with the interference effect progressively decreasing as the US preexposure interval was increased from 105 min to 3 days. No interference with conditioning occurred with preexposure intervals less than $105 \mathrm{~min}$.

A one-way analysis of variance computed on the amounts ingested during the postconditioning test indicated a significant effect of the various treatment conditions $[\mathrm{F}(8,83)=3.8, \mathrm{p}<.01]$. Subsequent comparisons with the Newman-Keuls test $(p<.05)$ showed that both Group 105 and Group No-Av drank significantly more saccharin than each of Groups TAC and 3-Day. Group 140 also drank more than Group 3-Day, but none of the other group differences were statistically significant. It is important to note that the greater test intakes of Groups 105 and 140 in comparison to Groups TAC and 3-Day cannot be attributed to differences in saccharin consumption during the conditioning trial, because these groups drank comparable amounts during conditioning.

\section{Discussion}

Consistent with previous findings (Domjan et al, 1977; Domjan \& Gregg, 1977), the present experiment indicates that exposure to a $1.0 \%$ saccharin solution $30 \mathrm{~min}$ after the injection of lithium chloride (Group TAC) results in less subsequent consumption of saccharin than exposure to this solution in the absence of drug administration (Group No-Av). If, in addition 
to the lithium treatment 30 min before saccharin exposure, subjects receive another lithium injection some time earlier, their aversion learning is disrupted. However, this interference effect occurs only when the US preexposure is given during a limited period before conditioning. Shorter preexposure intervals do not disrupt conditioning, and the interference effect is also attenuated as the US preexposure interval is increased beyond the optimal value, with no disruption of conditioning evident if the US preexposure occurs several days before conditioning.

The fact that US preexposure attenuated aversion learning only for a limited period suggests that the phenomenon reflects a transient aftereffect of the initial lithium injection. In this respect, the interference effect observed in the present study is similar to the previously documented effects of proximal US preexposure on aversions learned with more conventional forward conditioning procedures (Domjan \& Best, 1977). It appears that proximal US-preexposure effects are evident even if the nonpreexposed control group also receives its conditioning exposure to the CS flavor following drug injection.

Observation of the proximal US-preexposure effect in the present experiment not only serves to demonstrate the phenomenon with a new conditioning procedure, but also helps to elucidate its mechanisms. Since both preexposed and nonpreexposed subjects received the CS $30 \mathrm{~min}$ after drug injection, it is unlikely that they had the CS flavor differentially paired with recovery from malaise, as might have occurred in the Domjan and Best (1977) experiments. The temporal course of the interference effect observed in Experiment 1 also makes a generalization decrement interpretation less likely. If the preexposure US altered the perception of the CS and thereby contributed to a generalization decrement when subjects were subsequently tested in the normal state, then the greatest interference with performance would have been expected at the shortest preexposure intervals.

The present experiment was similar in many ways to the recent experiment of Best and Gemberling (1977) designed to determine the effects of proximal CS preexposure on flavor aversion learning. They systematically varied the interval between flavor preexposure and a subsequent flavor-lithium pairing and found that the maximum disruption of conditioning occurs when the CS preexposure is given about $4 \mathrm{~h}$ before conditioning, with shorter and longer preexposure intervals resulting in less interference.

The procedure used by Best and Gemberling (1977) involved two successive presentations of the CS flavor followed by an injection of lithium (CS-CS-Li), and the interval between the two CS exposures was varied. The present experiment involved a similar sequence of stimulus presentations ( $\mathrm{Li}$-Li-CS) and a similar manipulation, except that two successive lithium injections served in the place of the two CS exposures, and a single ex posure to the CS was given at the time Best anc Gemberling administered their single lithium injection Interestingly, the results obtained were also very similar As in the Best and Gemberling (1977) study, inter ference with aversion learning was greatest when a par ticular intermediate interval was used between the re peated stimulus presentations. with shorter and longe preexposure periods producing less disruption of con ditioning.

The congruence between the proximal CS-preexposure experiment reported by Best and Gemberling (1977), and the proximal US-preexposure experiment reported here indicates that less association occurs between two stimuli, $S_{1}$ and $S_{2}$, if training involves repetition of one 'of these events followed by the other $\left(S_{1}-S_{1}-S_{2}\right)$ than it the two events are presented only once in close tem. poral succession $\left(S_{1}-S_{2}\right)$. Furthermore, this relationship is observed both when $S_{1}$ is flavor exposure and $S_{2}$ is lithium injection as well as when the role of these events is reversed. Therefore, it is likely that the attenu. ated learning is produced by a mechanism which is activated by repetitions of a stimulus independent of the nature of the stimulus, be it a flavor or a drug injection.

\section{EXPERIMENT 2}

The proximal US-preexposure effect observed in Experiment 1 is similar to the previously observed effects of proximal US preexposure on aversions conditioned with drug treatment following taste exposure (Domjan \& Best, 1977) in that the interference with conditioning declined as the preexposure interval was increased beyond an optimal value. Another functional aspect of the effect of US preexposure on forward taste-aversion conditioning is that the aversion learning decreases as the dose of the US preexposure is increased (Cannon, Berman, Baker, \& Atkinson, 1975). Experiment 2 was designed to confirm that proximal US preexposure attenuates aversions learned as a result of postinjection taste exposure and to determine how this interference effect is influenced by the preexposure drug dose.

\section{Method}

Fifteen male and 45 female 50- to 60-day-old SpragueDawley rats born in the laboratory were used. Maintenance procedures were identical to those of Experiment 1 . Starting 9-11 days before conditioning, access to water was restricted to $30 \mathrm{~min}$ daily. The subjects were distributed among six groups $(\mathrm{Ns}=10)$ made as similar as possible with respect to weight and gender. During conditioning, cach subject received two intraperitoneal injections $105 \mathrm{~min}$ apart. Thirty minutes after the second injection, the subjects were given access to a $1.0 \%$ solution of sodium saccharin for $30 \mathrm{~min}$ or until they consumed $1 \mathrm{ml}$, whichever occurred first. For all subjects, the second intraperitoneal injection was $1.8 \mathrm{meq} / \mathrm{kg}$ of $.15 \mathrm{-M}$ lithium chloride. Independent groups received $.9,1.2,1.5,1.8$, and $3.0 \mathrm{meq} / \mathrm{kg}$ of .15-M lithium chloride in their first injection. The six th group of subjects did not receive such US preexposure. Instead of 


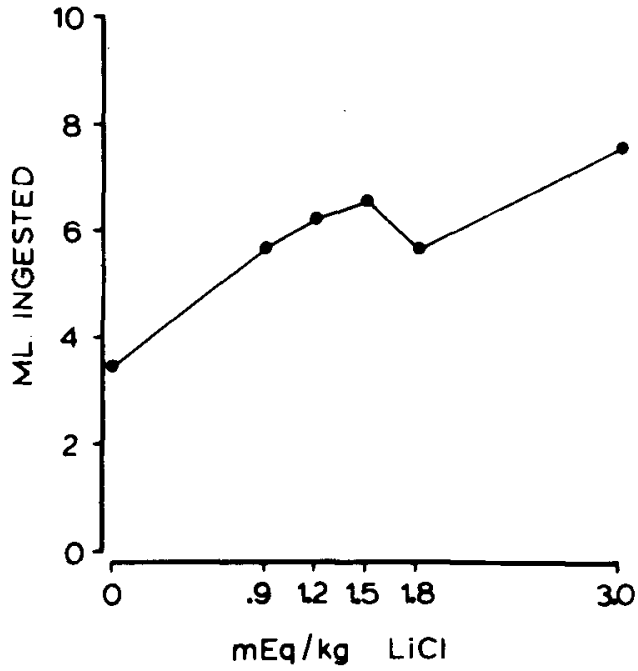

Figure 2. Mean intakes of saccharin several days after subjects received two lithium injections $105 \mathrm{~min}$ apart, followed $30 \mathrm{~min}$ later by exposure to saccharin. The dose of the first lithium injection was varied among independent groups; the second lithium injection was always $1.8 \mathrm{meq} / \mathrm{kg}$.

lithium, these rats were injected with $1.8 \mathrm{meq} / \mathrm{kg} .15 \cdot \mathrm{M}$ sodium chloride $105 \mathrm{~min}$ before their second injection. The daily $30-\mathrm{min}$ access to water was provided $2-3 \mathrm{~h}$ after the second injection. Six days after the conditioning trial, each subject received a 60 -min one-bottle test with the saccharin solution in the home cage following $23.5-\mathrm{h}$ water deprivation.

\section{Results and Discussion}

There were no significant group differences in saccharin intake during conditioning $(\mathrm{Ms}=.4 .6 \mathrm{ml})$. The amount of saccharin each group drank during the postconditioning test is summarized in Figure 2 as a function of lithium preexposure dose. As is evident from the figure, higher US-preexposure doses resulted in weaker taste aversions. This pattern of results yielded a significant linear trend between preexposure dose and consumption during the test session $[F(1,54)=6.00$, $\mathrm{p}<.05]$.

The present results confirm that proximal US preexposure can attenuate ingestional aversions which result from taste exposure after lithium treatment. Furthermore, the fact that weaker aversions were acquired by subjects which received higher dose preexposure injections shows that this effect of US preexposure is directly related to preexposure dose. In this respect, the proximal US-preexposure effect on aversions learned in a postinjection paradigm is similar to the deleterious effect of US preexposure on ingestional aversions learned with more conventional forward conditioning procedures (Cannon et al., 1975).

\section{EXPERIMENT 3}

Experiments 1 and 2 demonstrated that the effects of proximal US preexposure on aversions learned in a postinjection paradigm have temporal and dose-response characteristics which are similar to previously documented effects of US preexposure on forward tasteaversion conditioning. Experiment 3 was designed to compare directly the magnitude of the interference with conditioning which results from US preexposure in the postinjection and forward conditioning paradigms. As in Experiments 1 and 2, the procedure for US preexposure in the postinjection conditioning paradigm involved two successive lithium injections followed by exposure to saccharin ( $\mathrm{Li}-\mathrm{Li}-\mathrm{CS}$ ). In contrast, the procedure for US preexposure in the forward conditioning paradigm involved a lithium injection, followed by taste exposure and a second lithium injection (Li-CS-Li). To the extent that the interference with conditioning is determined entirely by repetition of lithium treatment with a particular interval between the two drug treatments, similar degrees of interference should be evident with the Li-Li-CS and the Li-CS-Li procedures. However, if the position of the CS flavor exposure in the stimulus sequence is also important, different degrees of interference may be evident with the two procedures.

\section{Method}

Forty-four male 50- to 60-day-old Sprague-Dawley rats from the Holtzman Co. (Madison, Wisconsin) were used with maintenance procedures identical to those of Experiment 1. Starting 8-9 days before conditioning, access to water was restricted to $30 \mathrm{~min}$ daily. The subjects were then assigned to four groups $(\mathrm{Ns}=11)$ of similar weight. On the conditioning day, each subject was allowed to drink $.25 \mathrm{ml}$ of a $1.0 \%$ solution of saccharin. In addition, each subject received two intraperitoneal injections $(12 \mathrm{meq} / \mathrm{kg})$.

For Group LiLiS, both injections on the conditioning day consisted of $.15 \mathrm{M}$ lithium chloride; the first injection was administered $105 \mathrm{~min}$ before the second lithium treatment, which occurred $30 \mathrm{~min}$ before saccharin exposure. Group NaLiS was treated the same way as Group LiLiS, except that its first injection consisted of $.15 \mathrm{M}$ sodium chloride. Group LiSLi received a lithium injection $65 \mathrm{~min}$ before saccharin access and a second lithium injection $45 \mathrm{~min}$ after the start of the saccharin exposure. Thus, the two lithium treatments for this group were separated by the same $105 \mathrm{~min}$ as occurred between the two lithium injections for Group LiLiS. The fourth group of subjects (NaSLi) was treated the same way as Group LiSLi, except that its first injection consisted of physiological saline instead of lithium.

On the conditioning day, the subjects were given their daily $30 \mathrm{~min}$ access to water $2.5 \mathrm{~h}$ after their last lithium injection. Water was also available for $30 \mathrm{~min}$ on the next day, and a 60 -min one-bottle test with the $1.0 \%$ saccharin solution was conducted the following day after $23.5 \mathrm{~h}$ water deprivation.

\section{Results}

Each subject drank the $.25 \mathrm{ml}$ of saccharin it was given on the conditioning day. Therefore, there were no group differences in saccharin consumption during conditioning. Cumulative intakes of saccharin during successive 15-min periods of the postconditioning test session are presented in Figure 3. Both US-preexposure groups (LiSLi and LiLiS) drank more than their respective nonpreexposed control groups ( $\mathrm{NaSLi}$ and $\mathrm{NaLiS}$ ). 


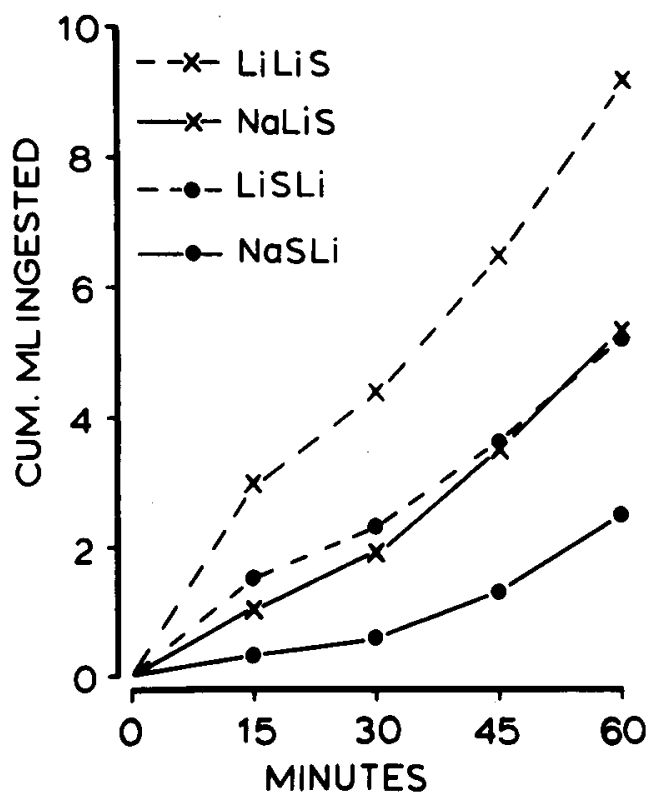

Figure 3. Mean cumulative intakes of saccharin several days after a treatment in which some subjects were exposed to saccharin either following two lithium injections (Group LiLiS) or between two lithium injections (Group LiSLi) administered 105 min apart. Groups NaLiS and NaSLi were treated the same way as Groups LiLiS and LiSLi, except that they were injected with physiological saline in place of the first lithium injection.

However, weaker aversions were learned as a result of exposure to saccharin after lithium treatment (Groups $\mathrm{NaLiS}$ and LiLiS) than if flavor exposure was given before a drug injection (Groups NaSLi and LiSLi).

The total amount consumed by each group during the test session was evaluated with an analysis of variance in which US preexposure and type of conditioning were treated as factorial variables. This analysis confirmed that US preexposure attenuated conditioning $[F(1,40)=8.6, p<.01]$ and that weaker aversions were learned with postinjection flavor exposure than with the more conventional forward conditioning procedure $[F(1,40)=9.1, p<.01]$. However, the interaction of the preexposure and conditioning variables was not significant $(F<1.0)$, indicating that proximal US preexposure did not have different effects on the two conditioning procedures.

\section{Discussion}

The present findings confirm that the deleterious effects of proximal US preexposure are not limited to the conventional conditioning procedure in which exposure to the CS flavor is followed by drug injection (Domjan \& Best, 1977). They also demonstrate that the degree of disruption of conditioning caused by proximal US preexposure is not different using the conventional forward conditioning procedure as compared to a conditioning procedure in which the $\mathrm{CS}$ is presented after drug injection. Whether or not the CS flavor was presented between the two lithium administrations for the US preexposed groups did not appear to influence the interference with conditioning. This outcome suggests that the interference with conditioning is not dependent on specifically when the CS is presented relative to the two US presentations. Rather, administration of the US shortly before its pairing with the CS flavor appears to be primarily responsible for the attenuation of conditioning.

It was noted in the introduction that if the proximal US preexposure effect is due to rehearsal of the first US presentation in short-term memory when the US is repeated (Wagner, 1976), then one might expect less interference with conditioning when a novel stimulus, such as the CS, is given between the two US administrations than if the novel CS is given after the two drug injections. The fact that the same degree of interference was observed in these two situations in the present experiment does not confirm this prediction. However, the present results can be easily reconciled with rehearsal theory by assuming that short-term memory has sufficient capacity so that presentation of the novel CS does not interfere with rehearsal of the preexposed US.

\section{EXPERIMENT 4}

Many aspects of the proximal CS-preexposure effect (Best \& Gemberling, 1977) and the proximal USpreexposure effect (Domjan \& Best, 1977, and the present studies) are consistent with the recently proposed stimulus rehearsal theory (Wagner, 1976). However, it is not clear from the theory why the interference effect does not occur with very short CS. preexposure (Best \& Gemberling, 1977) or US. preexposure (Experiment 1, present report) intervals. The disruption of conditioning produced by proximal CS or US preexposure is attributed to the prerepresentation or rehearsal of these stimuli in short-term memory, and this rehearsal of the preexposed stimuli is assumed to decrease with time. Therefore, the greatest disruption of conditioning would be expected with the shortest preexposure intervals, and conditioning should be an increasing monotonic function of the preexposure interval rather than a nonmonotonic function as was observed in Experiment 1 and the Best and Gemberling (1977) study.

Interference with conditioning may not be evident with very short CS- and US-preexposure intervals because under such circumstances the preexposure presentation of the CS or US can participate in the learned association. For example, US preexposure 30 or $70 \mathrm{~min}$ before the backward pairing of lithium and saccharin in Experiment 1 may not have attenuated aversion learning because the saccharin became associated with the preexposure lithium injection. Experiment 4 was designed to evaluate this possibility by testing not only the usual US-preexposed and nonpreexposed groups but also subjects given only the preexposure 
lithium injection before saccharin access. The third group was included to evaluate the extent to which saccharin could become associated with the preexposure lithium injection.

An additional objective of Experiment 4 was to determine whether a short US preexposure interval was as ineffective in disrupting forward taste- aversion conditioning as it was in disrupting aversions learned as a result of postinjection taste exposure in Experiment 1. Previous experiments have shown that proximal US preexposure disrupts forward taste-aversion conditioning if a 70-min interval is used between the repeated lithium injections (Domjan \& Best, 1977). However, a 70-min preexposure interval did not attenuate conditioning in a postinjection procedure in Experiment 1. These results suggest that with short preexposure intervals, US preexposure may have greater deleterious effects on aversions conditioned with post-CS lithium injections (Domjan \& Best, 1977) than aversions conditioned with pretaste drug treatment (Experiments 1-3).

\section{Method}

Fifty male 50- to 60-day-old Sprague-Dawley rats purchased from the ARS Sprague Dawley Co. (Madison, Wisconsin) were used with maintenance procedures identical to those of Experiment 1. Access to water was restricted to $30 \mathrm{~min}$ daily for the entire experiment, starting 8-9 days before conditioning. The subjects were assigned to six groups of similar weight. Three of these were comparison groups used to assess the effects of US preexposure on aversions resulting from postinjection flavor exposure, whereas the remaining three groups assessed the effects of US preexposure on aversions learned in a more conventional forward conditioning procedure.

During conditioning, each subject received access to a $1.0 \%$ solution of sodium saccharin for a maximum of $10 \mathrm{~min}$. Group Li30S $(N=8)$ received a single injection of lithium $30 \mathrm{~min}$ before the saccharin exposure. Group LiLiS $(\mathrm{N}=9)$ was treated the same way as Group Li30S except that it also received a preexposure injection of lithium $60 \mathrm{~min}$ before saccharin access. Thus, the two lithium injections for Group LiLiS occurred $30 \mathrm{~min}$ apart. The third postinjection comparison group, Li60S $(N=8)$, received only the preexposure injection of lithium $60 \mathrm{~min}$ before the saccharin presentation.

The forward taste-aversion conditioning control group, SLi $(\mathrm{N}=8)$, was injected with lithium $10 \mathrm{~min}$ after receiving access to saccharin. The forward conditioning US-preexposure group, LiSLi $(N=9)$, received the same postsaccharin injection as Group SLi. However, Group LiSLi was also injected with lithium $20 \mathrm{~min}$ before saccharin access. Therefore, the interval between the two lithium injections for Group LiSLi was $30 \mathrm{~min}$, as it was for the postinjection US-preexposure group, LiLiS. The final forward conditioning comparison group, Li20S $(N=8)$, received only the US-preexposure injection of lithium administered to Group $\mathrm{LiSLi}, 20 \mathrm{~min}$ before saccharin access.

Since Groups LiSLi and Li20S received saccharin exposure on the conditioning day $20 \mathrm{~min}$ after lithium treatment while all other groups received saccharin either without prior lithium injection (Group SLi) or a longer period ( 30 or $60 \mathrm{~min}$ ) after lithium treatment (Groups Li30S, LiLiS, and Li60S), intakes of saccharin were expected to be most suppressed in Groups $\mathrm{LiSLi}$ and $\mathrm{Li20S}$. Therefore, all of the other groups were allowed to drink only as much saccharin during conditioning as the mean intake of Groups LiSLi and Li20S $(.2 \mathrm{ml})$. This procedure precluded significant group differences in saccharin intake during conditioning.
The dose of all lithium injections was $1.8 \mathrm{meq} / \mathrm{kg}$ of a $.15-\mathrm{M}$ solution. On the conditioning day, the subjects received their daily 30 -min access to water $3-4 \mathrm{~h}$ after the saccharin exposure. On the $3 \mathrm{rd}$ and 5 th days after conditioning, each subject received a $120-\mathrm{min}$ one-bottle test with the saccharin solution following $23.5 \mathrm{~h}$ water deprivation. During each test, intakes were recorded at $15-\mathrm{min}$ intervals. On test days, the $30-\mathrm{min}$ maintenance water-access period was provided after the test session.

\section{Results}

Figure 4 summarizes the cumulative intakes during each test session for the forward conditioning comparison groups, SLi, LiSLi, and Li20S, while Figure 5 summarizes the test intakes of the postinjection comparison groups, Li30S, LiLiS, and Li60S. Both sets of groups drank very little saccharin during Test 1 . The postinjection comparison groups (Figure 5) drank more than the forward conditioning groups (Figure 4). However, there did not appear to be significant differences in intake among the three groups of each set. As expected, the subjects drank more during Test 2 than during Test 1 , and again the three postinjection comparison

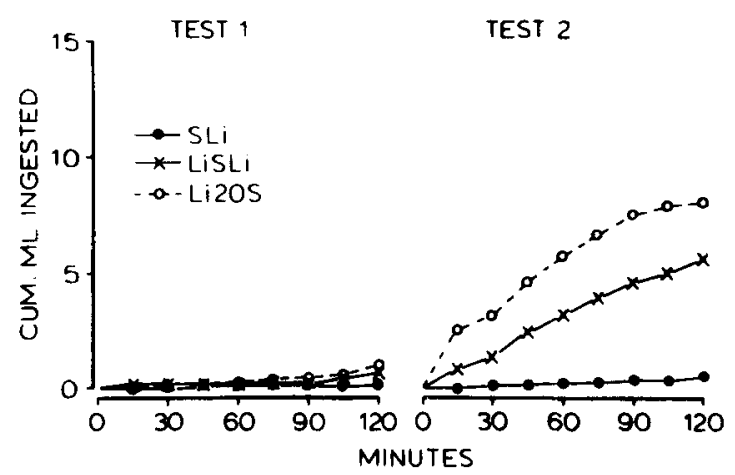

Figure 4. Mean cumulative intakes of saccharin during two test sessions following a treatment in which Group LiSLi was injected with lithium both before and after saccharin exposure, with a 30-min interval between the two injections; Group Li20S received only the first of the two injections given Group LiSLi, and Group SLi received only the second of these injections.

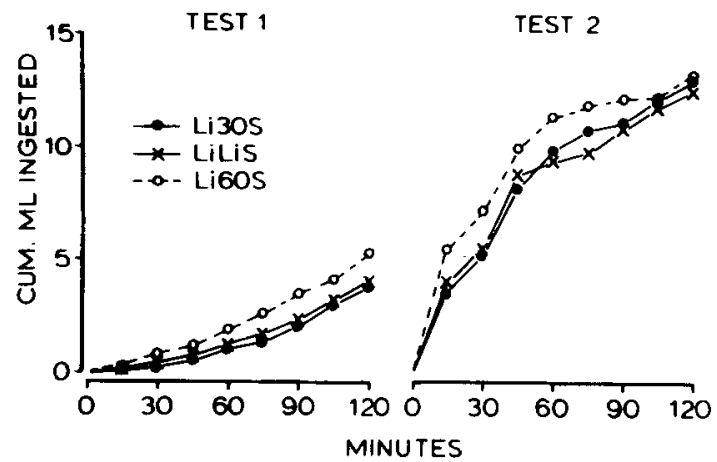

Figure 5. Mean cumulative intakes of saccharin during two test sessions following a treatment in which Group LiLiS received two lithium injections $30 \mathrm{~min}$ apart, followed $30 \mathrm{~min}$ later by exposure to saccharin; Group Li60S received only the first of the two injections given Group LiLiS, and Group Li30S received only the second of these injections. 
groups (Figure 5) consumed similar amounts. In contrast, the US-preexposed forward conditioned subjects (Group LiSLi) evidenced weaker aversions than the nonpreexposed Group SLi. Group Li20S also appeared to have learned a weaker aversion than Group SLi (Figure 4).

The total amount consumed during each test session was analyzed with a 2 by 3 analysis of variance involving the two conditioning procedures (forward vs. postinjection) and the three groups compared in each conditioning paradigm. For Test 1 , the only significant effect revealed by this analysis was that the postinjection groups drank more saccharin than the forward conditioned groups $[F(1,44)=34.2, p<.01]$. There was also a significant effect of conditioning paradigm in Test 2 $[F(1,44)=66.6, p<.01]$. In addition, for Test 2 there was a significant Groups by Conditioning Paradigm interaction $[F(2,44)=4.8, p<.05]$. The Neuman-Keuls test $(p<.05)$ was then used to assess group differences in Test 2 intake for each conditioning paradigm. No significant differences were found among the postinjection comparison groups. In contrast, the forward conditioned aversion control Group SLi evidenced significantly stronger aversions than each of Groups LiSLi and Li20S, which did not differ significantly from each other.

\section{Discussion}

The present results confirm the finding in Experiment 1 that with a short (i.e., $30-\mathrm{min}$ ) preexposure interval, US preexposure does not attenuate taste aversions learned as a result of postinjection flavor exposure (Group LiLiS vs. Group Li30S). In contrast, when the same 30 -min interval was used between successive lithium injections, a significant interference effect of US preexposure was observed on aversions learned in a more conventional forward conditioning procedure (Group LiSLi vs. Group SLi). These results indicate that with short preexposure intervals, the effects of US preexposure are more evident in forward conditioning paradigms than if aversions are conditioned with postinjection taste exposure.

The fact that an interference effect was more evident in forward conditioning than with aversions learned in a postinjection procedure may encourage speculation that different mechanisms mediate the US preexposure effect in the two conditioning paradigms. However, such considerations should not ignore striking similarities in the pattern of results obtained in the two situations. In each set of comparison groups, subjects that received two lithium injections (Groups LiSLi and LiLiS) did not learn significantly stronger aversions than corresponding subjects which received only the first of these two lithium treatments (Groups Li20S and Li60S, respectively). Thus, both Groups LiSLi and LiLiS behaved as if their second lithium in. jection had no effect, despite the fact that the second injection conditioned a significant aversion when administered by itself (Groups SLi and Li30S). The absence of an observable effect of the second lithium injection in Groups LiSLi and LiLiS is unlikely to have been due to a floor effect, because at least one group of subjects (SLi) drank less during the test sessions than Groups LiSLi and LiLiS. These considerations suggest that the first lithium injection substantially reduced the impact of the second injection in both Groups LiSLi and LiLiS. Such an outcome is rather remarkable considering that the two injections were administered only $30 \mathrm{~min}$ apart. Given such a short interval, one might have expected to observe summation of the effects of two injections rather than the absence of an effect of the second injection.

If, as the above discussion suggests, the first lithium injection in Group LiLiS reduced the effectiveness of the second injection, then why did Group LiLiS not learn a weaker taste aversion than the nonpreexposed control group, Li30S? The absence of the usual USpreexposure effect in the comparison of Groups LiLiS and Li30S was probably due to the strong conditioning of a taste aversion by the preexposure lithium treatment. With the brief preexposure interval used, the USpreexposure injection was given $60 \mathrm{~min}$ before saccharin exposure. Previous research has shown that exposure to $1.0 \%$ saccharin $60 \mathrm{~min}$ after lithium treatment results in a conditioned aversion to the saccharin flavor (Domjan \& Gregg, 1977). Consistent with this observation, in the present experiment, lithium treatment $60 \mathrm{~min}$ before saccharin exposure (Group Li60S) resulted in as strong an aversion to the saccharin solution as lithium treatment $30 \mathrm{~min}$ before saccharin access (Group Li30S). This aversion conditioning induced by the proximal administration of the preexposure injection made it impossible to observe weaker aversions in the preexposed (LiLiS) than in the nonpreexposed (Li30S) groups. In contrast, among the forward conditioning comparison groups, the preexposure injection did not result in as strong an aversion as that observed in the nonpreexposed subjects (Groups $\mathrm{Li} 20 \mathrm{~S}$ vs. SLi). Therefore, attenuations in conditioning produced by the preexposure injection (Group LiSLi) could be more evident.

It is interesting to note that the above analysis of the absence of attenuated aversion learning in a postinjection paradigm following US preexposure at short preexposure intervals is similar to a suggested explanation for the similar absence of disrupted conditioning with short CS-preexposure intervals (Best \& Gemberling, 1977). Short CS-preexposure intervals probably permit the entry of the preexposure presentation of the CS into association with the US and thereby prevent observation of an interference with processing of the second CS presentation. Apparently, the deleterious effects of stimulus preexposure on the processing of subsequently presented CS or US events can be 
observed only if the preexposure presentation of the CS or US does not itself participate to a great extent in the association to be established.

\section{GENERAL DISCUSSION}

Previous research has shown that exposure to a concentrated saccharin solution within $60 \mathrm{~min}$ after the injection of lithium chloride results in a conditioned aversion to the saccharin flavor (Domjan \& Gregg, 1977; Domjan et al., 1977). The present experiments demonstrate that such aversion learning can be disrupted if subjects also receive an additional lithium injection some time earlier (Experiments 1-3). This interference effect of US preexposure is directly related to the dose of the preexposure drug injection (Experiment 2) and is a decreasing function of the preexposure interval, beyond the optimal interval for observing the phenom. enon (Experiment 1). Very short preexposure intervals (e.g., $30 \mathrm{~min}$ ) do not disrupt learning (Experiments 1 and 4), probably because under these circumstances the preexposure injection itself conditions as strong an aversion as that evident in nonpreexposed subjects (Experiment 4).

The present experiments differed from most previously published reports on the deleterious effects of US preexposure in that only one preconditioning injection of the drug was administered and the interference effect was evident only if this US exposure occurred less than $24 \mathrm{~h}$ before the conditioning trial. Most previous studies have involved repeated preconditioning exposures administered several days before conditioning (see reviews by Braveman, 1977; Gamzu, 1977). The temporary nature of the interference effect observed in the present experiments contrasts with the more durable preexposure effects documented in these earlier reports and cannot be explained by the various associative interference mechanisms that have been proposed (e.g., Mikulka, Leard, \& Klein, 1977; Rudy, Iwens, \& Best, 1977) because these mechanisms are not assumed to exhibit temporal decay.

The preexposure effect observed in the postinjection conditioning paradigm in the present experiments is similar in many ways to the proximal US-preexposure effect recently demonstrated in the forward conditioning of ingestional aversions (Cannon et al., 1975; Domjan \& Best, 1977) and eyeblink responses (Terry, 1976). The magnitude of the interference at moderate preexposure intervals is comparable in the two conditioning procedures (Experiment 3), and in both paradigms the effects of drug pretreatment are similarly determined by the preexposure drug dose and the preexposure interval (Experiments 1 and 2; Cannon et al., 1975; Domjan \& Best, 1977). The effect of proximal US preexposure on the two conditioning pro. cedures was different only if a very short preexposure interval was used (Experiment 4). However, even in this situation, preexposed subjects, which received two drug injections, responded as if they had received only the first injection. Thus, the first injection appeared to have blocked the effectiveness of the second injection regard. less of the type of conditioning procedure used.

The similarity of the effects of proximal US preexposure on aversions learned as a result of taste exposure following drug treatment as compared to aversions learned with more conventional forward conditioning procedures would have limited theoretical significance if the postinjection conditioning procedure used were functionally the same as forward conditioning. One might suggest that exposure to a flavor after drug injec. tion is the same as a forward taste-injection pairing, because the malaise induced by the injection does not begin until after the taste exposure in both cases. How. ever, such an analysis is inapplicable to the postinjection conditioning procedure used in the present experiments. In each study, the interval between lithium and subsequent taste exposure was $30 \mathrm{~min}$. Previous research has shown that intraperitoneal injections of lithium suppress saccharin (Barker \& Smith, 1974; Domjan, 1977) and sometimes water (Barker \& Smith, 1974) consumption, starting less than $15 \mathrm{~min}$ after the drug treatment. These findings indicate that the malaise induced by lithium has a latency much shorter than $30 \mathrm{~min}$. Furthermore, if a 30 -min injection-taste interval results in a forward pairing of taste with illness, all of the various tastes which are easily conditioned in forward conditioning paradigms also should be condition. able with backward injection-taste pairings. However, in contrast to their rapid associability with toxicosis in forward conditioning paradigms, weak concentrations of saccharin (e.g., .1\% saccharin) do not become conditioned if they are presented more than $10 \mathrm{~min}$ after lithium injection (Barker \& Smith, 1974; Domjan \& Gregg, 1977). Aversion conditioning to flavors presented longer periods after drug treatment appears to be limited to more concentrated taste solutions (Domjan \& Gregg, 1977). Finally, the fact that the deleterious effects of US preexposure at very short preexposure intervals are more observable with forward conditioning procedures than with a postinjection taste exposure procedure (Experiment 4) provides additional evidence of the difference between these two conditioning paradigms.

Given the difference between the postinjection aversion conditioning procedure and the more conventional forward conditioning procedure, the present experiments serve to extend the generality of the proximal US preexposure phenomenon to a new situation. The present experiments also demonstrate that the interference effect is not restricted to situations in which the flavor CS is presented between two drug injections (Domjan \& Best, 1977), but also occurs if the flavor CS is presented following the two injections. Therefore, explanations of the effect which focus on presentation of only 
the preexposure US shortly before the CS (as occurs in forward conditioning) are not generally applicable.

Since the effects of US preexposure at moderate preexposure intervals appear to be independent of when the CS is presented relative to the repeated US exposures, but are influenced by the interval between the iterated USs, explanations which focus on the role of the US-US interval would seem most promising. Two prominent recently proposed theories are of this nature. Both attribute the proximal US preexposure phenomenon to a reduction in the effectiveness of the second US caused by the preexposure US presentation. Wagner's (1976) rehearsal theory assumes that attenuations of the second US occur because the second US is presented while the subject is rehearsing the first US presentation in short-term memory. In contrast, Solomon and Corbit's (1974) theory assumes that the effectiveness of the second US is reduced because the second US is presented during an opponent process which is stimulated by the first US presentation. Both theories predict less interference as the interval between the iterated US presentations is increased, because both rehearsal induced by the first US and the opponent process stimulated by the first US are assumed to decay with time.

\section{REFERENCES}

Barker, L. M., \& Smith, J. C. A comparison of taste aversions induced by radiation and lithium chloride in CS-US and US-CS paradigms. Journal of Comparative and Physiological Psychology, 1974, 87, 644-654.

Best, M. R., \& Gemberling, G. A. Role of short-term processes in the conditioned stimulus preexposure effect and the delay of reinforcement gradient in long-delay taste-aversion learning. Journal of Experimental Psychology: Animal Behavior Processes, 1977, 3, 253-263.

Braveman, N. S. What studies on preexposure to pharmacological agents tell us about the nature of the aversion-inducing treatment. In L. M. Barker, M. R. Best, \& $M$. Domjan (Eds.), Learning mechanisms in food selection. Waco, Texas: Baylor University Press, 1977.

Cannon, D. S., Berman, R. F., Baker, T. B., \& Atrinson, C. A. Effect of preconditioning unconditioned stimulus experience on learned taste aversions. Journal of Experimental Psychology: Animal Behavior Processes, 1975, 1, 270-284.

Cappell. H., \& LeBlanc, A. E. Conditioned aversion by amphetamine: Rates of acquisition and loss of the attenuating effects of prior exposure. Psychopharmacologia, 1975, 43, $157 \cdot 162$.
Domjan, M. CS preexposure in taste-aversion learning: Effects of deprivation and preexposure duration. Leaming and Motivation, 1972. 3. 389-402.

Domjan, M. Selective suppression of drinking during a limited period following aversive drug treatment in rats. Jourtal of Experimental Psychology: Animal Behavior Processes. 1977. 3. $66-76$.

Domjan, M.. \& Best, M. R. Paradoxical effects of proximal unconditioned stimulus preexposure: Interference with and conditioning of a taste aversion. Journal of Experimental Psychology: Animal Behavior Processes, 1977, 3, 310-321.

Domjan, M.. \& Gregg, B. Long-delay backward taste-aversion conditioning with lithium. Physiology and Behavior, 1977. 18. 59-62.

Doman, M., Schorr, R., \& Best, M. R. Early environmental influences on conditioned and unconditioned ingestional and locomotor behaviors. Developmental Psychobiology. 1977, 10, 499-506.

GAMZU, E. The multifaceted nature of taste-aversion-inducing agents: Is there a single common factor? In L. M. Barker. M. R. Best, \& M. Domjan (Eds.), Learning mechanisms in food selection. Waco. Texas: Baylor University Press. 1977.

Lubow. R. E. Latent inhibition. Psychological Bulletin, 1973. 79. 398-407.

Mikulka, P. J.. Leard, B., \& Klein, S. B. Illness-alone exposure as a source of interference with the acquisition and retention of a taste aversion. Journal of Experimental Psychology: Animal Behavior Processes, 1977, 3. 189-201.

Riley. A. L.. Jacobs. W. J., \& LoLordo, V. M. Drug exposure and acquisition and retention of a conditioned taste aversion. Journal of Comparative and Physiological Psychology. 1976. 90, 799-807.

Rudy, J. W., Iwens, J., \& Best. P. J. Pairing novel exteroceptive cues and illness reduces illness-induced taste aversions. Journal of Experimental Psychology: Animal Behavior Processes, 1977, 3, 14.25.

Siegel, S., \& Domjan, M. Backward conditioning as an inhibitory procedure. Learning and Motivation, 1971, 2, 1-11.

Solomon, R. L., \& Corbit, J. D. An opponent-process theory of motivation: I. Temporal dynamics of affect. Psychological Review, 1974, 81, 119-145.

TERRY, W. S. Effects of priming unconditioned stimulus representation in short-term memory on Pavlovian conditioning. Journal of Experimental Psychology: Animal Behavior Processes, $1976,2.354-369$.

Vogel. J. R., \& Nathan, B. A. Reduction of learned taste aversion by preexposure to drugs. Psychopharmacologia, 1976. 49. $167 \cdot 172$.

WAGner, A. R. Priming in STM: An information processing mechanism for self-generated or retrieval-generated depression in performance. In T. J. Tighe \& R. N. Leaton (Eds.). Habituation: Perspectives from child development, animal behavior, and neurophysiology. Hillsdale, N.J: Erlbaum. 1976.

(Received for publication November 17. 1977; revision accepted January 13, 1978.) 